\title{
Component simulation in problems of calculated model formation of automatic machine mechanisms
}

\author{
Igor Telegin ${ }^{1, *}$, Alexander Kozlov ${ }^{1}$ and Alexander Zhirkov ${ }^{2}$ \\ ${ }^{1}$ Lipetsk State Technical University, 398600, 30, Moskovskaya St., Lipetsk, Russia \\ ${ }^{2}$ Bryansk State Technical University, 241035, 7, Bulvar 50-letiya Oktyabrya, Bryansk, Russia
}

\begin{abstract}
The paper deals with the problems of the component simulation method application in the problems of the automation of the mechanical system model formation with the further possibility of their CAD-realization. The purpose of the investigations mentioned consists in the automation of the CAD-model formation of high-speed mechanisms in automatic machines and in the analysis of dynamic processes occurred in their units taking into account their elasto-inertial properties, power dissipation, gaps in kinematic pairs, friction forces, design and technological loads. As an example in the paper there are considered a formalization of stages in the computer model formation of the cutting mechanism in cold stamping automatic machine AV1818 and methods of for the computation of their parameters on the basis of its solid-state model.
\end{abstract}

One of the basic problems in the field of production and modernization of automatic machines, in particular, multi-position cold pressing automatic machines (CAM) or, a more general name of press machines is the increase of reliability, productivity and durability.

The analysis of reasons in automatic machine failures shows that in the overwhelming majority of cases in the ground are dynamic (oscillating) processes the intensity of which increases sharply with the increase of speed modes in equipment operation [1 -9]. When operating the pressing machines with the productivity from fifty up to some hundred products per minute the most substantial influence upon such parameters as reliability and durability is carried out by dynamic processes developing in auxiliary systems: of a blank transfer between technological positions, cutting and others to the accurate positioning of their executive units are made particularly strict requirements [10 - 13].

Current methods of the dynamic phenomena account at designing mechanisms consist in the substitution of the latter by some abstract designs models. But, in order that the model reflected really dynamic processes in a real mechanism it is necessary that the structure itself and a mathematical description should be developed theoretically correct and substantiated and quantitative characteristics of its parameters should be defined with the sufficient degree of accuracy.

The investigations of dynamic processes in mechanisms on the basis of models allowed taking into account elasto-inertial characteristics of their units give a possibility already at the first stage of designing to determine not only loads in these units and their kinematic parameters, but to carry out numerical experiments for the determination a maximum productivity and to determine efficient speed modes for a mechanism operation in particular and an automatic machine as a whole [13-15].

The investigation of the mechanic system dynamics is:

- the formation of the successive approximation file: real mechanism - dynamic model - mathematical model - simulator numerical realization - computer model;

- the development of the method for a quantitative and qualitative definition of the level of dynamic processes occurred in the mechanism and affecting its reliability and working capacity under the influence of outer factors (operating speed, technological forces) and changes of inner parameters (kinematic pair wear, structure loads, adjustment parameters).

The described above procedure of a dynamic mechanism analysis is known $[1,4,11]$. But, in spite of the constant improvement connected with the mass (complexity) number increase, with the account of gaps in kinematic connections, with the specification of elastic tie characteristics, with dissipation parameters, friction forces, external loads it did not gain a wide dissemination. Basic reasons of this were as follows:

- low degree of accuracy and a large labor intensity in the computation of model parameters, in the first place, elastic-inertial properties;

- large labor intensity of works connected with the development of a dynamic model of a specific mechanism, its mathematic description and a program of realization;

- impossibility of the assessment of authenticity of results obtained without experimental investigation fulfillment [13-15].

\footnotetext{
* Corresponding author: vv.telegin@yandex.ru
} 
The purpose in the development of the procedure of dynamic processes analysis in mechanisms consists in the decrease of labor intensity in dynamic investigations of mechanisms and increase of their credibility. At the heart of it there are methods of component modeling and object-oriented techniques [2, 7, 16], and also the methods of solid-state modeling [14, 15].

The mechanism can be considered as a totality of some elements connected between each other by different ties. By means of these ties the mechanism elements interact with each other. With the term 'element' we will identify a separately taken part of the mechanism, its certain fragment or, vice versa, some parts simultaneously. This definition of an element corresponds to the 'object' concept - one of basic in the theory of a component modeling of complex dynamic systems and object-oriented techniques $[2,13-15,16]$.

For the visual presentation of the information on mechanisms there are used diagrams (schematic circuits, kinematics, and others) $2 \mathrm{D}$ and $3 \mathrm{D}$ drawings and also dynamic models. To the point all these mechanism presentations (not only the latest ones) are at the same time their models of different complexity and purpose. Evidently, the object can contain both a single part or even its element and some parts. Any processes in such an object can be modeled in any way and, in accordance with the principles of encapsulation and polymorphism, at the given stage their nature and mathematical-, program-, or physical description are not under consideration.

Such an approach is of interest both at the solution of problems connected with the development of dynamic and mathematical models of mechanisms and, what is particularly significant, their program realizations for the further computer processing $[2,13,16]$.

Let us consider a stepwise process in the formation of the object presentation of a cutting mechanism in cold pressing automatic machine AB1818 and its mathematical description. At each stage of this process is carried out an addition of a new unit to the mechanism, to its object presentation - new object, and to the mathematical description - response of the unit joined.
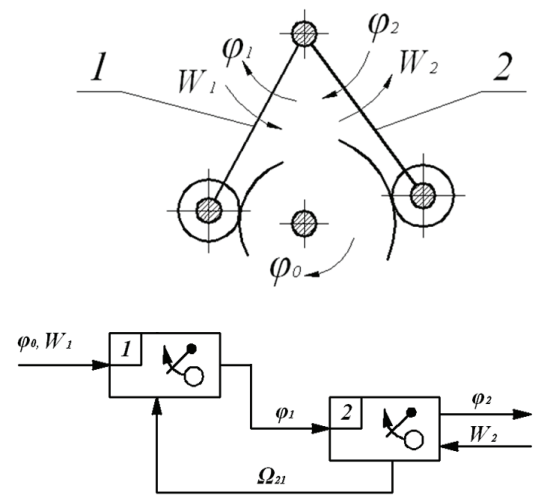

Fig. 1. Cam mechanism and its object model

The formation of mathematical models of the equations of motion (1) - (9) is shown in Figures 1, 2 and 3 and carried out in the assumption that each object is modeled by a single-mass oscillating system described by a differential equation of the second order. Certainly, a real model and its mathematical description may be of any complexity.

Mathematical model (Fig. 1):

$$
\left\{\begin{array}{l}
J_{1} \ddot{\varphi}_{1}=-c_{1}\left(\varphi_{1}-U_{1}\right)-b_{1}\left(\dot{\varphi}_{1}-\dot{U}_{1}\right)+W_{1}+\Omega_{21} \\
J_{2} \ddot{\varphi}_{2}=-c_{3}\left(\varphi_{2}-\varphi_{1}\right)-b_{3}\left(\dot{\varphi}_{2}-\dot{\varphi}_{1}\right)+W_{2}
\end{array}\right.
$$

Where external load:

$$
\begin{gathered}
W_{1}=-M_{m p 1}-M_{z a m}, \\
W_{2}=-M_{m p 2}+M_{z a m}-c_{2}\left(\varphi_{2}-U_{2}\right)-b_{2}\left(\dot{\varphi}_{2}-\dot{U}_{2}\right) .
\end{gathered}
$$

Disturbance:

$$
\Omega_{21}=c_{3}\left(\varphi_{2}-\varphi_{1}\right)+b_{3}\left(\dot{\varphi}_{2}-\dot{\varphi}_{1}\right) .
$$
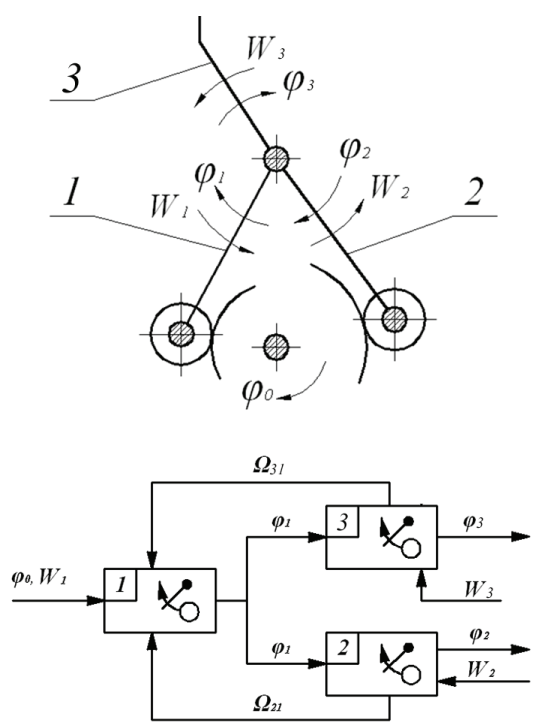

Fig. 2. Cutting mechanism without a knife rod and its object model
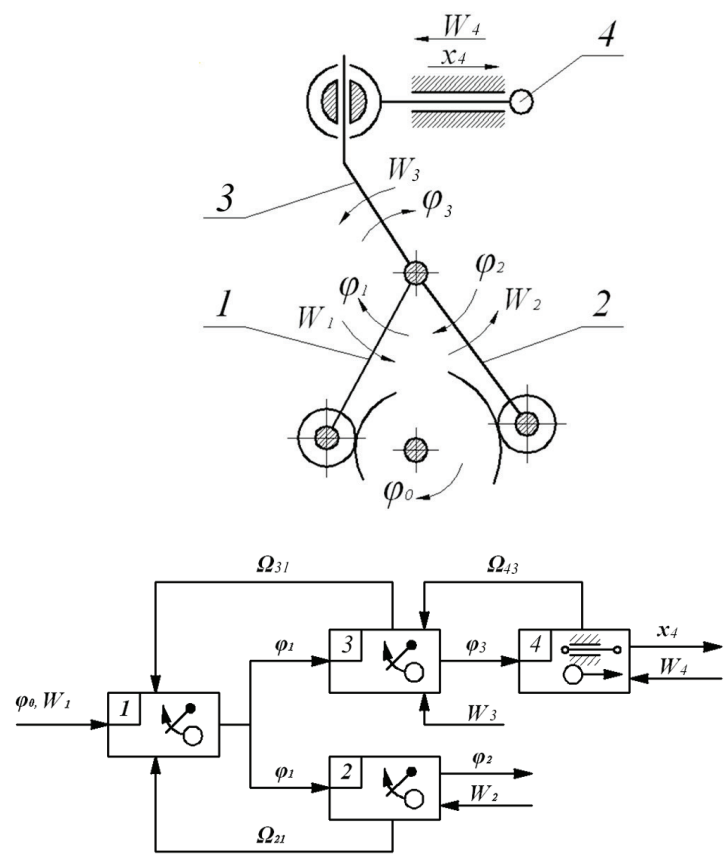

Fig. 3. Mechanism of cutting and its object model 
Mathematical model (Fig. 2):

$\left\{\begin{array}{l}J_{1} \ddot{\varphi}_{1}=-c_{1}\left(\varphi_{1}-U_{1}\right)-b_{1}\left(\dot{\varphi}_{1}-\dot{U}_{1}\right)+W_{1}+\Omega_{21}+\Omega_{31} \\ J_{2} \ddot{\varphi}_{2}=-c_{12}\left(\varphi_{2}-\varphi_{1}\right)-b_{12}\left(\dot{\varphi}_{2}-\dot{\varphi}_{1}\right)+W_{2} \\ J_{3} \ddot{\varphi}_{3}=-c_{3}\left(\varphi_{3}-\varphi_{1}\right)-b_{3}\left(\dot{\varphi}_{3}-\dot{\varphi}_{1}\right)+W_{3}\end{array}\right.$

Where external load:

$$
W_{3}=-M_{m p 3} .
$$

Disturbance:

$$
\Omega_{31}=\left(c_{3}\left(\varphi_{3}-U_{3}\right)+b_{3}\left(\dot{\varphi}_{3}-\dot{U}_{3}\right)\right) U_{3}^{\prime} .
$$

Mathematical model (Fig. 3):

$$
\left\{\begin{array}{l}
J_{1} \ddot{\varphi}_{1}=-c_{1}\left(\varphi_{1}-U_{1}\right)-b_{1}\left(\dot{\varphi}_{1}-\dot{U}_{1}\right)+W_{1}+\Omega_{21}+\Omega_{31} \\
J_{2} \ddot{\varphi}_{2}=-c_{12}\left(\varphi_{2}-\varphi_{1}\right)-b_{12}\left(\dot{\varphi}_{2}-\dot{\varphi}_{1}\right)+W_{2} \\
J_{3} \ddot{\varphi}_{3}=-c_{3}\left(\varphi_{3}-\varphi_{1}\right)-b_{3}\left(\dot{\varphi}_{3}-\dot{\varphi}_{1}\right)+W_{3}+\Omega_{43} \\
m_{4} \ddot{x}_{4}=-c_{4}\left(x_{4}-U_{4}\right)-b_{4}\left(\dot{x}_{4}-\dot{U}_{4}\right)+W_{4}
\end{array}\right.
$$

External load:

$$
W_{4}=-P_{m p 4}-P_{m e x},
$$

Disturbance:

$$
\Omega_{43}=\left(c_{4}\left(x_{4}-U_{4}\right)+b_{4}\left(\dot{x}_{4}-\dot{U}_{4}\right)\right) U_{4}^{\prime} .
$$

In Figures $1-3$ through positions 1 and 2 there are designated levers of a forward and reverse stroke of the cutting mechanism (Fig. 4), 3 - lever connecting levers 1 and 2 with knife rod $4 . \varphi_{0}$ - rotation angle of cams rotating at constant speed $\varphi_{1}, \varphi_{2}, \varphi_{3}, x_{4}$ - rotation angles of levers and a linear displacement of a knife rod, W1 ... W4 - external loads $(2,5,8)$ affecting units of the mechanism (friction forces, technological force, spring pressing force $\left(M_{\text {zam }}\right)$ ) with rigidity, $c_{12}$ connecting levers 1 and 2.

In equations (1), (4), (7) $J_{1}, J_{2}, J_{3}$ and $\mathrm{m} 4$ moments of inertia and a mass of corresponding units, $c_{1}$ $\ldots c_{4}$ - rigidity, $b_{1} \ldots b_{4}$ - parameters of dissipation, $U_{1}$ ... $U_{4}$ - functions of the position of mechanism units. Impacts upon a current object of other objects (3), (6), (9) connected with it are designated as $\Omega_{21}, \Omega_{31}, \Omega_{43}$.

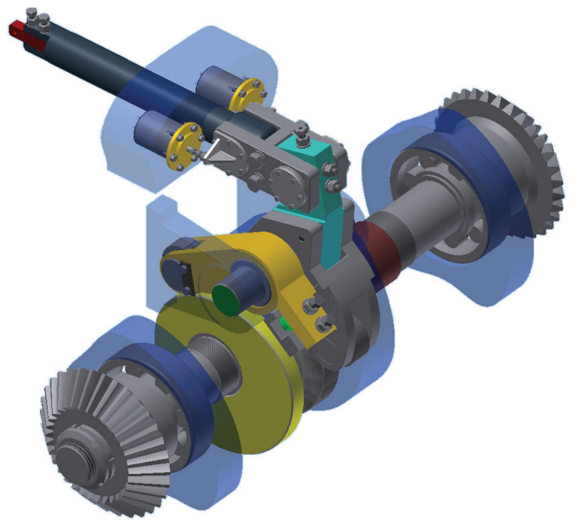

Fig. 4. Cutting mechanism KhVA AV1918 (3D-model).
It should be particularly emphasized that a set of objects presenting a mechanism (object presentation) and a mechanism itself (Fig. 4) are adequate each other. The substitution of reality with a model is carried out only at the level of an object. In such a way, the accuracy of dynamic investigation results is defined with 'goodness' of those objects used in these investigations.

And there is one more very significant circumstance. A possibility to create own user's objects out of already available 'reliable' objects having no care regarding the matter of adequacy [13].

The solution of problems connected with the formation of object-oriented models, with the computation of its parameters and the fulfillment of dynamic investigations is automated. The complex of corresponding programs is combined into a united system - dam (dynamic analysis of mechanisms). The present application gives wide potentialities connected with the control of input parameters of the mechanism, a computation process, the results of computations and their visualization $[13-15]$.

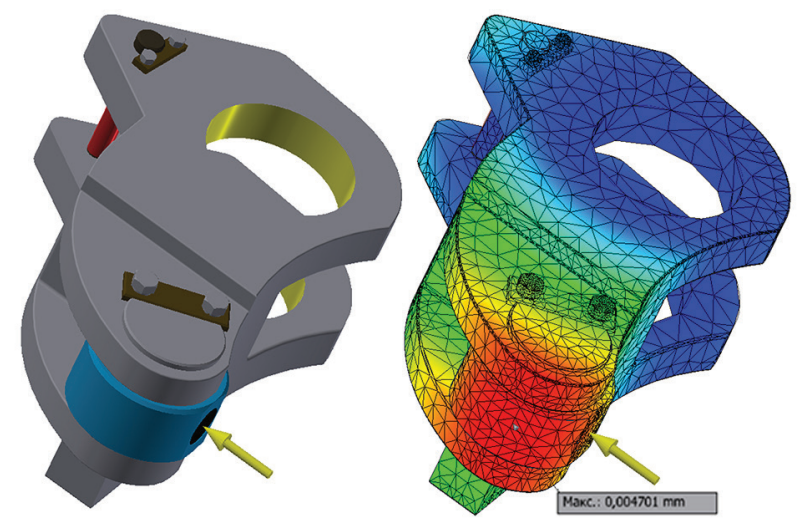

Fig. 5. Rigidity computation for the direct motion lever

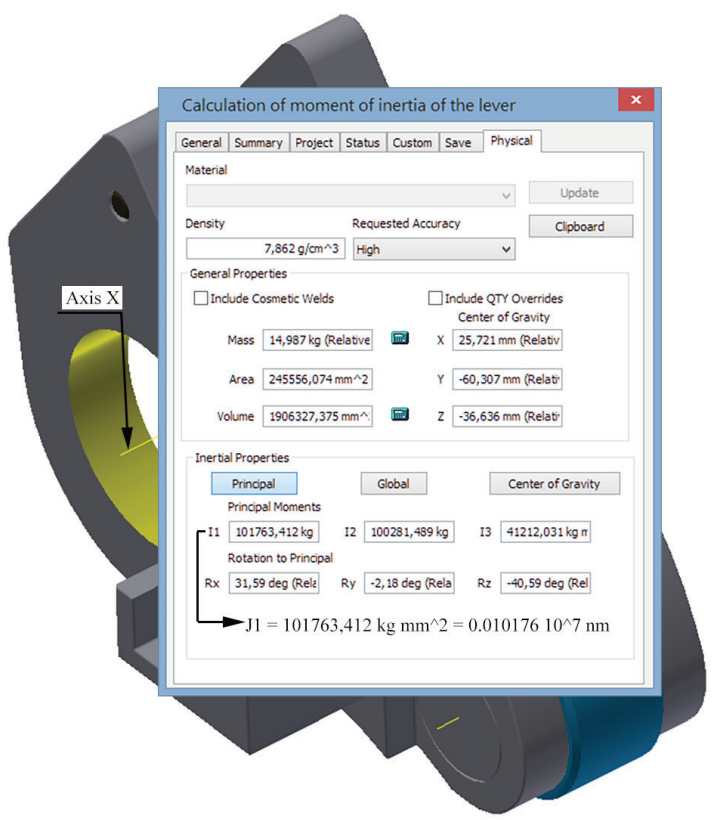

Fig. 6. Computation of the inertia moment for the direct motion lever 
Current methods of 3D-modeling including modules of the finite-measuring analysis allow calculating with sufficiently high accuracy elastic-inertial characteristics (rigidity, inertia moments and mass) constituting mechanism models $[14,15]$. To the number of programs allowing the solution of similar problems belongs Autodesk Inventor Professional in the environment of which there is created a solid-state model of the mechanism shown in Figure 4.

In Figure 5 there is shown a procedure for the computation of rigidity for the lever of direct motion. The deformable elements of the lever are loaded with force of $1000 \mathrm{~N}$, a radius of reference point rotation is equal to $200 \mathrm{~mm}(160-$ lever $+40-$ roller $)$. The reference point displacement under the influence of moment of force equal to $200 \mathrm{~N} \cdot \mathrm{m}-0.004701 \mathrm{~mm}$ or $0.23505 \cdot 10^{-4} \mathrm{rad}$. Lever rigidity $-0.859 \cdot 10^{7} \mathrm{~N} \cdot \mathrm{m} / \mathrm{rad}$.

In Figure 6 there is shown a procedure for the definition of unit inertia characteristics, in this case, a moment of inertia regarding the axis of coinciding with its axis of rotation.

The combined application of the solid-state modeling systems with the module of a finite element method (FEM) and the system of a dynamic analysis developed on the basis of the material of this paper allows carrying out dynamic investigations simultaneously with static computations of strength and works connected with the effectiveness increase of mechanisms under development.

The results were obtained within of the state assignment of the Ministry of Education and Science of Russia (project No. 11.9505.2017/8.9).

\section{References}

1. I.I. Wolfson, Oscillations of Machines with Mechanisms of Cyclic Action (1990)

2. J. Rumbaugh, Object-Oriented Modeling and Design (1991)

3. N.I. Levitsky, Machine and Mechanism Theory (1979)

4. V.L. Veits, M.Z. Kolovsky, A.E. Kochura, Dynamics of controlled machine aggregates (1984)

5. Vibrations in Engineering: Reference Book, 3, (1980)

6. I.A. Krivosheev, D.A. Akhmetzyanov, Modeling dynamic processes in a complex system (2003)

7. V.M. Ponyatsky, E.N. Davydov, V.G. Fedorischeva, A.V. Osadchiy, IV Kislinsky, General and applied mechanics, Bulletin of the Nizhny Novgorod University. N.I. Lobachevsky, 4 (2), 283-284, (2011)

8. A.M. Kozlov, E.V. Kiryushchenko, Bulletin of Ufa State Aircraft Technical University, 4, 117-123, (2012)

9. A.M. Kozlov, A.A. Kozlov, Russian Engineering Research, 29(7), 743-746 (2009).

10. Forging and stamping: Reference Book, 3, (1987)
11. Yu.A. Miropolsky, Cold Volumetric Stamping on Automatic Machines (2001)

12. Vibrations in Engineering: Reference Book, 5, (1981)

13. V.V. Telegin, Proceeding of Samara Scientific Center of the RAS, 12, 4(3), 623-628 (2010)

14. V.V. Telegin, Proceedings of Samara Scientific Center of the RAS, 14, 4(5), 1306-1309, (2012)

15. V.V. Telegin, Proceedings of TulSU. Serie. Mechanics of Deformable Solid and Metal Forming, 1, 197-206, (2004)

16. E.S. Benkovsky, Yu.B. Kolesov, Yu.B. Senichenkov, Dynamic System Practical Modeling (2002) 